\title{
Electric Field-Induced Surface Melting of Gold Observed In Situ at Room Temperature and at Atomic Resolution Using TEM
}

Ludvig de Knoop $^{1 *}$, Mikael Juhani Kuisma ${ }^{2}$, Joakim Löfgren ${ }^{1}$, Kristof Lodewijks ${ }^{1}$, Mattias Thuvander ${ }^{1}$, Paul Erhart ${ }^{1}$, Alexandre Dmitriev ${ }^{3}$ and Eva Olsson ${ }^{1 *}$

1. Chalmers University of Technology, Department of Physics, Gothenburg, Sweden.

2. University of Jyväskylä, Department of Chemistry, Jyväskylä, Finland.

3. University of Gothenburg, Department of Physics, Gothenburg, Sweden.

* Corresponding authors: ludvig.deknoop@chalmers.se and eva.olsson@chalmers.se

It is well known that an elevated temperature can induce surface melting and surface roughening of metals [1]. Apart from elevated temperatures, also intense electric fields can alter the surface state, e.g. as in field-assisted ionization used in field ion microscopy or as in field evaporation, utilized in atom probe tomography. However, as far as we know, there are no atomically resolved observations of metal surfaces at fields just below the field-evaporation field. Here, we have used in situ transmission electron microscopy (TEM) to study the surface of a gold nanocone while simultaneously applying an electric field at room temperature [Fig. 1(a)-(b)]. At electric fields of around $25 \mathrm{~V} / \mathrm{nm}$ we observed a surface roughening, or disorder, of the outmost 2-4 atomic layers [Fig. 1(d)-(e)]. By reducing the applied electric field, the disordered atomic layers reversed to the original and crystalline structure. In other words, the process is reversible. The inset in Fig. 1(c) and (d) show the fast Fourier transformation (FFT) during the phase transformation obtained from the very apex of the nanocone. An FFT typical for a crystalline structure can be seen in (c), while the FFT in (d) features a ring, which could be indicative of the disordered or surface melted state. By increasing the electric field further, the atoms field evaporated, at around $30 \mathrm{~V} / \mathrm{nm}$ [Fig. 1(e)-(f)] [2].

Extensive $a b$ initio molecular dynamics simulations were made in order to understand the mechanism behind the disordered surface. The simulations showed that the observed phenomenon could be attributed, not to an increase in temperature from the electric field, but, to a vanishing energy cost to form surface defects at intense electric fields. In Fig. 2(a), gold nanoparticles as a function of temperature and electric field can be seen. The effective surface area as a function of electric field of the nanoparticles at $360 \mathrm{~K}$ from Fig. 2(a) can be seen in Fig. 2(b). Depending on changes in surface area, three different stages were extracted, corresponding to a normal (crystalline), surface roughened (disordered or surface melted) and field evaporated phase. Figure 2(c) show the mean square displacement (MSD) as a function of the electric field and the temperature. As can be seen, an electric field of $25 \mathrm{~V} / \mathrm{nm}$ (the onset for the disordered phase), corresponds to a relative change of around $15 \%$, at a temperature of $300 \mathrm{~K}$ (orange curve). For the field-free case (the blue linear slope), $15 \%$ increase of the MSD corresponds to only around $50 \mathrm{~K}$ temperature increase. In order words, this was not sufficient to explain the observed disordered or surface melted phase. Instead, it was found to be energetically more favorable to introduce surface defects in intense electric fields. The temperature increase from the electron beam was calculated using the Arrhenius equation and was estimated to be $16 \pm 4 \mathrm{~K}$, which originating in a 7 times higher diffusion rate of the $\mathrm{Au}$ atoms under the electron beam [2].

The ability to reversibly control the surface structure at the atomic level using an electric field could open up research fields in low-dimensional phases of matter as well as for new applications in catalysis, sensors and field-effect transistor technology. 
References:

[1] J. W. M. Frenken and J. F. van der Veen, Phys. Rev. Lett. 54 (1985), p. 134.

[2] L de Knoop et al., Physical Review Materials 2 (2018), p. 085006.

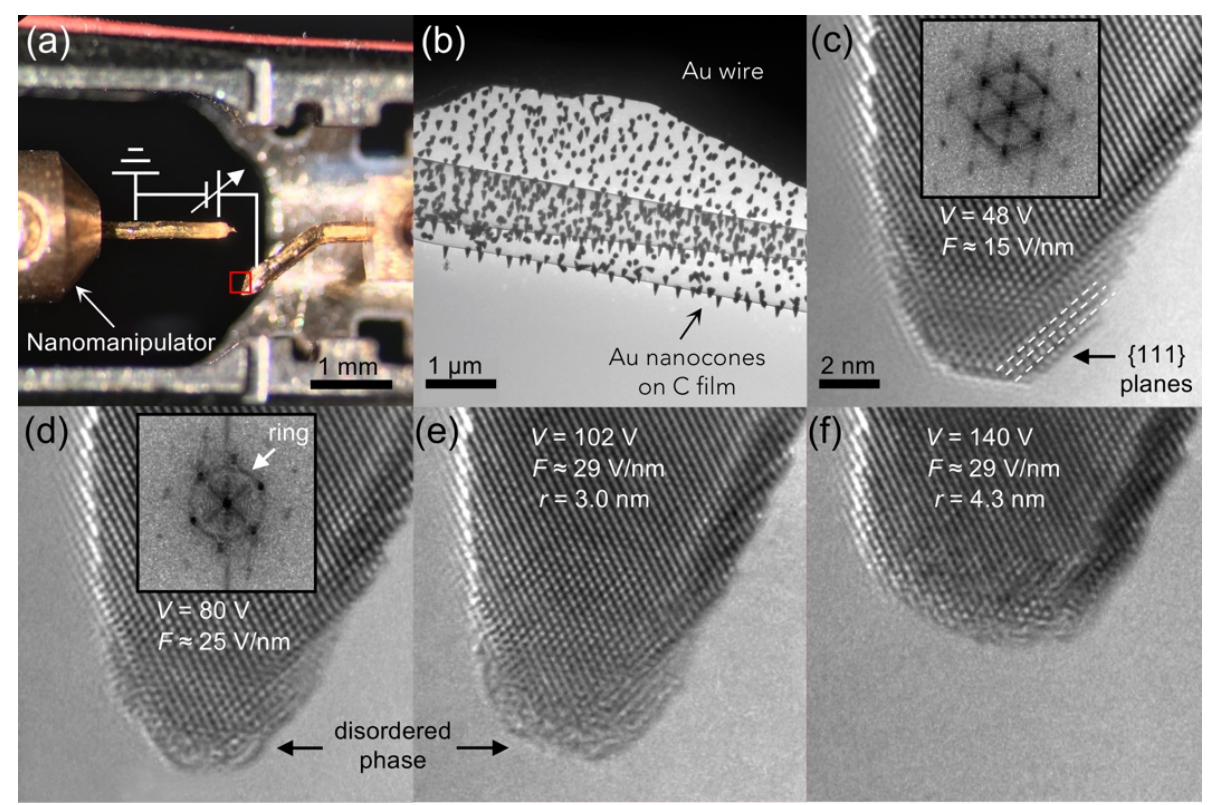

Figure 1. (a) Front part of the in situ TEM holder showing the nanomanipulator and sample with an electrical circuit schematic overlaid. The red square indicates the area displayed in (b). (b)-(f) TEM micrographs, in (b) of Au nanocones on a $\mathrm{C}$ film. (c) At an electric field of $\mathrm{F} \approx 15 \mathrm{~V} / \mathrm{nm}$, the nanocone rests in a crystalline state (inset shows the fast Fourier transformation (FFT)). In (d), at F $\approx 25 \mathrm{~V} / \mathrm{nm}$ the outmost atomic layers have switched to a disordered or surface melted phase. The FFT displays a ring, indicative of a disordered phase. By increasing the field further, the atoms are ionized and evaporated, starting in (e) and ending in (f) [2]. The scale bar in (c) also applies for (d)-(f).

(a)

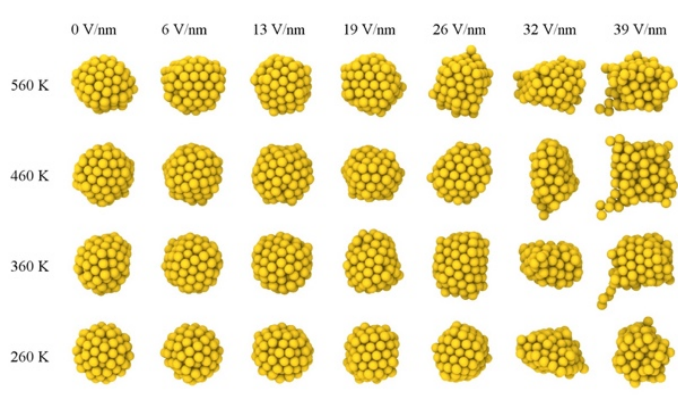

(b)

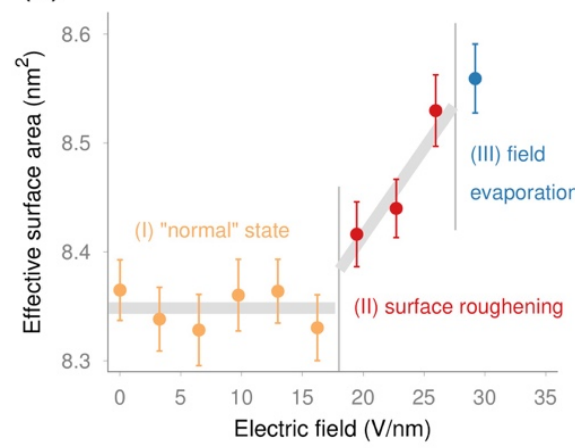

(c) Temperature (K) 300350400450500550600

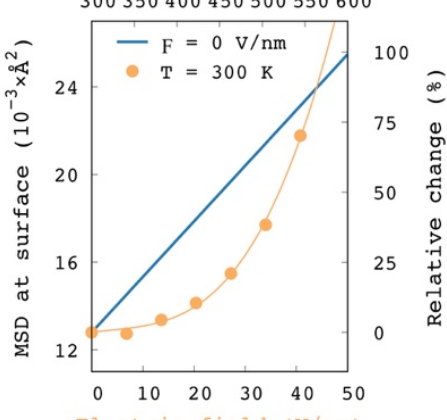

Figure 2. Ab initio molecular dynamics simulations of nanoparticles. In (a), the simulated nanoparticles as a function of temperature and electric field can be seen. (b) shows the effective surface area of the nanoparticles as a function of the electric field (based on (a) at $360 \mathrm{~K}$ ) with three stages visible; normal (crystalline), surface roughening (disordering or surface melting) and the field evaporation phase. The mean square displacement (MSD) as function of temperature and electric field can be seen in (c) [2]. 